\title{
Omphalolith: a rare umbilical concretion
}

\section{Siham Mansouri, Sara Mai, Badr Hassam, Meriem Meziane}

\author{
Dermatology Department, University Hospital Ibn Sina, Rabat Morocco
}

Corresponding author: Dr. Siham Mansouri, E-mail: siham-mnsr@hotmail.fr

Sir,

A 76-year-old woman was referred by her general physician to our dermatology department for the management of suspicious looking umbilical nodule of 10 years duration. She had no abdominal pain, weight loss, or fever. The patient did not give any history of passage of mucous, faces, or other discharge through the umbilicus. On exam she had a painful brownish-black, dry appearing mass of approximately 2 centimeters in length (Fig. 1) protruding from her umbilicus. There was no frank ulceration. The dermoscopic examination did not show a pigmented network or vascular patterns. It exhibited a dry crusted appearance. (Fig. 2). The remainder of the examination was unremarkable. Clinically, an omphalolith or umbilical concretion was suspected. It was easily removed with a warmed glycerin preparation. The histopathological examination of the specimen revealed concentric lamellae of keratin without evidence of any tumor.

Omphalolith is an uncommon entity under normal circumstances, generally asymptomatic, very few cases have been reported. It refers to sebum and keratin that have accumulated into a stone-like concretion in an umbilical cleft [1]. This calculi may remain undiagnosed for many years until revealed by secondary complication (infection or ulceration) [1,2]. Omphalolith generally presents with a firm, black umbilical mass that could be mistaken for other benign or malignant tumors of the umbilicus (melanoma, primary umbilical malignancy, umbilical metastasis, cholesteatoma, endometriosis...) $[3,4]$, pushing for unnecessary and costly consultations, procedures and imaging studies [5]. Superficial parts of the omphalolith can appear dark brown or black, likely due to melanin and oxidation of lipids much like an open comedone [4]. Omphalolith is usually associated with bad hygiene, when the patient fails to clean a deep umbilical cleft enough to contain a significant

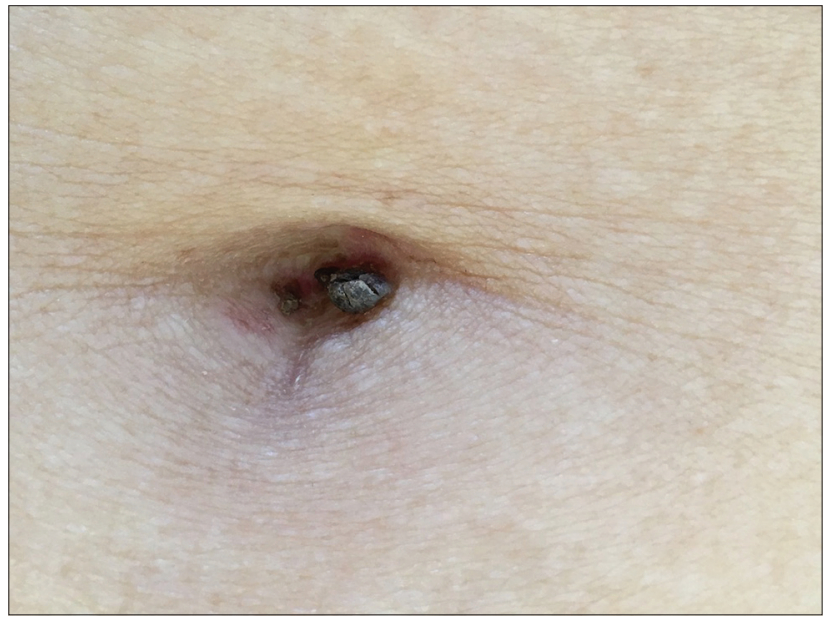

Figure 1: A painful brownish-black umbilical mass.

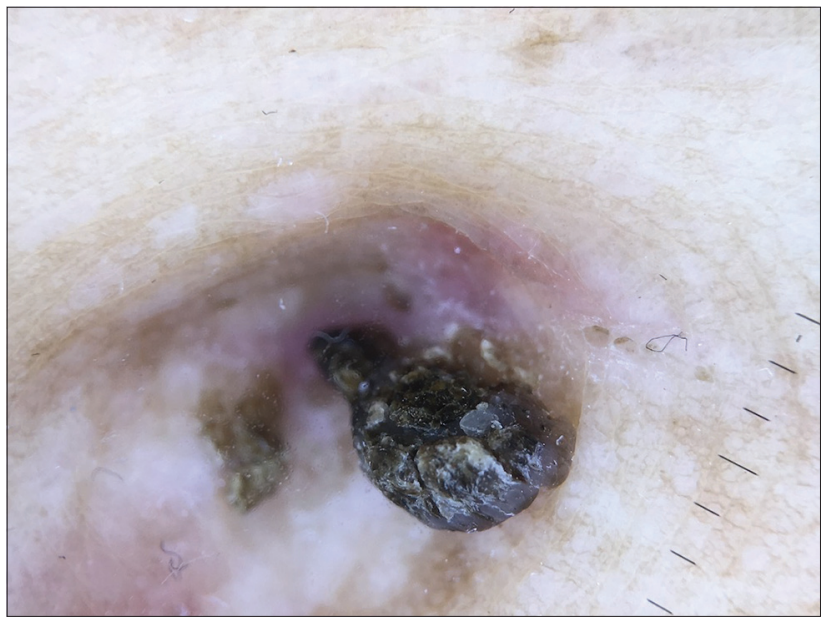

Figure 2: The dermoscopic examination exhibited a dry crusted appearance

concretion [3]. It can be removed by gentle manipulation or through adequate opening of umbilical foramen under local anesthesia to the periumbilical area.

Recognition of the diagnosis of omphalolith is important owing to the rarity of the condition. It's

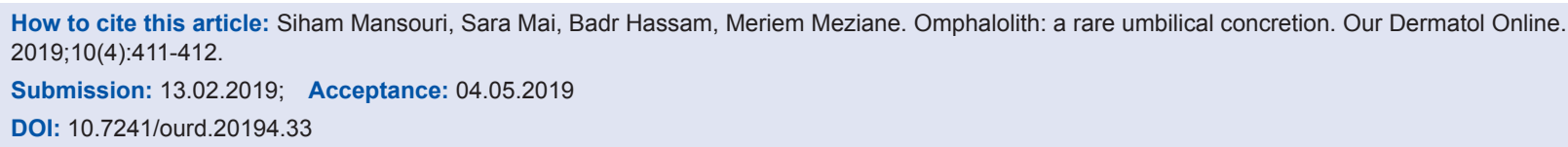


crucial to differentiate it from other benign and malignant conditions of the umbilicus and to avoid unnecessary imaging and procedures.

\section{Consent}

The examination of the patient was conducted according to the Declaration of Helsinki principles.

\section{REFERENCES}

1. Ichiki Y, Kitajima Y. Omphalith. Clin Exp Dermatol. 2008;34:420-1.

2. Mihara K. Omphalolith: An umbilical concretion to recognize.
J Gen Intern Med. 2016;31:1396.

3. Nittala P. Omphalolith/umbolith: CT and MR imaging appearances in two patients. Singapore Med J. 2009;50:745.

4. Sheehan D, Sarwat H, Vijayaraghavan G. Umbilical concretion. Radiol Case. 2011;5:25-31.

5. Kumar SKL, Reddy CO, Reddy K. Omphalolith. Indian J Surg. 2011;73:238-9.

Copyright by Siham Mansouri, et al. This is an open-access article distributed under the terms of the Creative Commons Attribution License, which permits unrestricted use, distribution, and reproduction in any medium, provided the original author and source are credited.

Source of Support: Nil, Conflict of Interest: None declared. 